\title{
ANTECEDENTES SOBRE LA CREACIÓN DE LA ESCUELA DE GRADUADOS Y PERFECCIONAMIENTO PROFESIONAL EN LA UNIVERSIDAD DE CONCEPCIÓN*
}

\author{
BACKGROUND ON THE CREATION OF THE GRADUATE \\ AND PROFESSIONAL CONTINUING EDUCATION SCHOOL \\ AT UNIVERSIDAD DE CONCEPCION
}

\author{
DANNY GONZALO MONSÁLVEZ ARANEDA**
}

Resumen: El presente trabajo tiene como objetivo dar cuenta de uno de los aspectos académicos más trascendentes de lo que ha sido la historia de la Universidad de Concepción en sus más de cien años de vida. Nos estamos refiriendo al impulso y apoyo otorgado a sus docentes para que estos iniciaran estudios de perfeccionamiento en el extranjero, así como la contratación de destacados académicos e investigadores nacionales e internacionales, con el objetivo de reforzar la docencia en la casa de estudios. Dicho proceso trajo como consecuencia la inquietud intelectual y el interés profesional por parte de algunos académicos de impulsar cursos de posgrado y de perfeccionamiento en la Universidad, proceso que se institucionalizó a comienzos de los años setenta, cuando bajo la Rectoría de Edgardo Enríquez, se decidió concretar la creación de una Escuela de Graduados y Perfeccionamiento Profesional, destinada a reforzar el trabajo científico de sus académicas y académicos, y al mismo tiempo profundizar la docencia e investigación del más alto nivel.

Palabras Clave: Universidad de Concepción, Escuela de Graduados y Perfeccionamiento Profesional, Edgardo Enríquez.

Aвstract: The purpose of this paper is to highlight one of the most transcendental academic moments in the history of the Universidad de Concepcion in its more than 100 years of existence. We are referring to the encouragement and support granted to their faculty to pursue further studies abroad, and the process of hiring outstanding foreign and local academics and researchers with the purpose of strengthening the teaching processes of this university. Such events ignited the intellectual restlessness and professional interest of some academics to promote and create post-graduate courses and also professional continuing education programs. This process was institutionalized in the early 1960's under the administration of rector Edgardo Enriquez, when the Graduate

\footnotetext{
* El presente texto se enmarca en el Proyecto Multidisciplinario "Ciencia, desarrollo y sociedad. Acercamiento a la producción de saberes pluridisciplinares en la Universidad de Concepción”, código 219.064.050-M, del cual el autor es investigador responsable.

** Doctor en Historia. Departamento de Historia, Universidad de Concepción, Chile. Investigador del Programa Ciencia, Desarrollo y Sociedad en América Latina, de la Universidad de Concepción (CIDESAL). Correo electrónico: dannymonsalvez@udec.cl. Orcid: https://orcid.org/0000-0001-7132-5468.
} 
and Professional Continuing Education School was created. Its aim is to strengthen the scientific work of its faculty as well as improving teaching and research at the highest level.

Keywords: Universidad de Concepción, Graduate and Professional Continuing Education School, Edgardo Enriquez.

Recibido: 2020-06-12. Aceptado: 2020-11-03.

\section{INTRODUCCIÓN}

T A INFORMACIÓN Y DOCUMENTACIÓN sobre los estudios de posgrado en Lla Universidad de Concepción se encuentra dispersa en carpetas y estantes del Archivo Central de la casa de estudios, es decir, no existe al día de hoy un texto que logre sintetizar o reunir los antecedentes históricos sobre lo que han sido los derroteros de la docencia de posgrado, así como la creación de la que durante varios años se llamó Escuela de Graduados y hoy recibe el nombre de Dirección de Posgrado. En otras palabras, la historia de la Universidad de Concepción en sus ya más de cien años de vida, todavía mantiene una deuda con su historia, más aun por la importancia que ha tenido y tiene en nuestros días. En vista de esto, el presente trabajo se ha planteado como objetivo central dar a conocer aquellos antecedentes generales y fundamentales sobre los orígenes de los estudios de perfeccionamiento y posgrado en la Universidad de Concepción, así como la gestación de la Escuela de Graduados y Perfeccionamiento Profesional.

Si bien la información que hemos reunido al día de hoy nos permite construir un texto que aborda con bastante claridad todo ese proceso, en las siguientes líneas solo daremos cuenta de una parte menor de una investigación mayor en curso. De esta forma, nos hacemos cargo, desde la disciplina histórica, del vacío que al día de hoy tenemos con una de las áreas más importantes con las cuales cuenta la Universidad de Concepción, como son sus estudios de posgrado, ya que es una de las casas de estudios líderes en materia de posgrado a nivel nacional y la universidad del centro sur de Chile con mayor número de posgrados.

\section{ALGUNOS ANTECEDENTES}

Desde su fundación, en 1919, la Universidad de Concepción ha brindado apoyo a sus académicos y académicas para que emprendan viajes al extran- 
jero con el objetivo de perfeccionar y complementar su formación profesional, académica y científica. Así, una generación significativa de jóvenes ha estado en contacto con grandes centros universitarios, ya sea de Europa o de Estados Unidos. En todo este proceso fue importante la contratación, en la década de 1920 de algunos renombrados investigadores europeos que muy pronto se convirtieron en la cabeza de equipos de investigación y cuyas clases se transformaron en una profundización de la ciencia, lo cual sin lugar a dudas fue la base real y un antecedente claro de la preocupación por el perfeccionamiento académico a través de cursos de posgrado en la Universidad.

Paralelamente a las estadías y visitas que realizaba el personal universitario en el exterior, se impulsó en ciertas unidades académicas de la Universidad la idea de crear algunos cursos denominados de posgraduados, que tuvieran como objetivo entregar una determinada profundización en ciertas áreas del conocimiento científico, proceso que se extendió hasta mediados del siglo XX, cuando, a partir de la década del cincuenta, la idea de avanzar en la creación de programas de posgrado y de una Escuela de Graduados tomará fuerza al interior de la casa de estudios.

Por ejemplo, en octubre de 1952 se desarrolló el primer congreso de académicos, estudiantes y administrativos de la Universidad. En dicho congreso se planteó, entre otras cosas, la viabilidad de un Doctorado en Ciencias, cursos de posgraduados y Doctorado en Matemática, Física, Química e Ingeniería Química y Doctorado en Ciencias Biológicas (La Patria, 23, 24 y 25 de octubre de 1952). A lo anterior se sumó el acuerdo suscrito para solicitar al Consejo y Directorio de la Universidad que formara una Comisión que avanzara en la creación de un Doctorado en Ciencias, programa que contaría con el apoyo de las facultades y de los profesores Gustavo Pizarro de la Escuela de Ingeniería y Bruno Günther de la Escuela de Medicina (ver “Una comisión...," La Patria, 25 de octubre de 1952, p. 9).

Cuatro años más tarde, en 1956, asumía como Rector de la Universidad, el abogado David Stitchkin, quien impulsó, con el apoyo de la Unesco, un plan de reestructuración académica en la casa de estudios, destinado a la creación de cuatro institutos centrales: Biología, Química, Matemática y Física.

Uno de los aspectos señalados por los asesores de la Unesco fue el de las debilidades en la formación y contenidos de algunas disciplinas, el caso específico de Química, acotando que una de las tareas inmediatas era impulsar el perfeccionamiento de los docentes de lo que sería el futuro Instituto Central de Química, es decir, la especialización a través de programas 
de posgrado tanto en el ámbito de los propios institutos, de acuerdo al reglamento de los Institutos Centrales en su artículo $2^{\circ}$ letra b) -"Promover doctorados en su campo en base a planes de estudio o programas desarrollados por él y aprobados por el H. Consejo Universitario" (Universidad de Concepción, 11 de mayo de 1959, p. 2)-, como del incentivo a profesores del Instituto para que viajaran al extranjero a seguir estudios de posgrado, básicamente destinados a la obtención del grado de doctor (p. 2).

Otro momento importante aconteció durante el rectorado de Ignacio González Ginouvés (1962-1968). Bajo su mandato, el año 1963, se preparó un anteproyecto de reforma docente, el cual en 1964 recibió el respaldo del Consejo y Directorio de la Universidad. Ese mismo año, concurrió a Concepción por dos meses el experto de la Unesco, Joseph Lauwerys, quien estuvo a cargo del estudio del proyecto, el cual fue difundido y analizado en toda la comunidad universitaria: "Por último, el Directorio y el Consejo aprobaron los planes finales, en conformidad al Proyecto elaborado por la Misión Lauwerys a fines de Octubre de 1964, y acordaron iniciar las reforma en el año académico de 1965” (González, 1968, p. 29). Uno de los puntos más significativos de dicha reforma apuntaba a "Crear los estudios de graduados" (p. 31). Durante el rectorado de Ignacio González se establecieron los grados académicos de Bachiller, Licenciado y Doctor, e incluso se aprobó la creación y organización de la Escuela de Graduados; sin embargo, esta no se concretó en aquellos años, y hubo que esperar hasta la rectoría de Edgardo Enríquez para conseguirlo (González, 1968, pp. 31 y 38).

Ahora bien, la idea de crear una Escuela de Graduados la encontramos en la convención de exalumnos de la Universidad de Concepción, celebrada los días 22 y 23 de abril de 1967, instancia en la cual la Mesa Directiva de esta agrupación dio a conocer al Rector Ignacio González las conclusiones de dicho encuentro, de las cuales una de las más importantes era la necesidad de contar con una Escuela de Graduados: “(...) anhelo de numerosos ex alumnos por alcanzar, a través de una profundización, especialización e investigación de un problema determinado, algún grado académico" (Universidad de Concepción, 8 de mayo de 1967). Esta Escuela de Graduados estaría destinada a:

$1^{\circ}$ - los alumnos que hayan obtenido el primer grado académico o BACHILLER;

$2^{\circ}$ - otorgar grados académicos de LICENCIADO (segundo grado) y de DOCTOR (tercer grado), y

$3^{\circ}$ - eventualmente, a preocuparse de ofrecer estudios postdoctorales. (p. 1) 
La creación de esta Escuela de Graduados debía tener como propósito contribuir a la formación científica de los alumnos de la Universidad de Concepción, a través de una instrucción avanzada en las ciencias, las letras y las artes. Para esto era necesario contar con una "(...) estrecha colaboración entre profesores y alumnos y en función de las necesidades del desarrollo económico social del país", lo cual posteriormente debería conducir “(...) a la formación de verdaderos dirigentes en el campo de las ciencias, las letras, las artes y de la sociedad toda" (Universidad de Concepción, 8 de mayo de 1967, p. 2).

Finalmente, la sociedad de exalumnos de la Universidad de Concepción dejó estipulada

(...) su urgente y preferente preocupación por crear la escuela de graduados, respondiendo con ello a necesidades creadas, primero, por la reforma docente impulsada por la propia Universidad y, segundo, para satisfacer sentidas aspiraciones de un gran número de miembros de esta sociedad. (Universidad de Concepción, 8 de mayo de 1967, p. 2).

\section{EL RECTORADO DE EDGARDO ENRÍQUEZ FRÖDDEN Y LA CREACIÓN DE LA ESCUELA DE GRADUADOS Y PERFECCIONAMIENTO PROFESIONAL}

Fue bajo la rectoría de Edgardo Enríquez (1969-1972) que comenzó a tomar fuerza la idea de crear una Escuela de Graduados en la Universidad de Concepción. Pero fue específicamente el vicerrector Galo Gómez Oyarzún quien recogió los antiguos anhelos e impulsó dicho proceso. Así, con fecha 2 de julio de 1971, Galo Gómez designó una subcomisión destinada a estudiar y proponer los fundamentos y estructura de una Escuela que tuviera a su cargo la Docencia de Postgrado. Se designó Presidente y Secretario a los profesores Ennio Vivaldi y Mario Pozo, respectivamente (Universidad de Concepción, septiembre de 1971).

La subcomisión trabajó durante el segundo semestre del año 1971 en la elaboración de un texto base para la futura institucionalización de una Escuela de Graduados y Perfeccionamiento Profesional. En aquellas reuniones se analizaron las variables que debían conducir a la Universidad al establecimiento de una institución que albergara los estudios de posgrado en la casa de estudios. Además, se designó a los profesores Ennio Vivaldi, Pablo Dobud y René Labraña para que presentaran un resumen del proyec- 
to al Consejo Superior (Informe creación de la Escuela de Graduados y de la Escuela de Perfeccionamiento Profesional de la Universidad de Concepción, Concepción, 1972, p. 2).

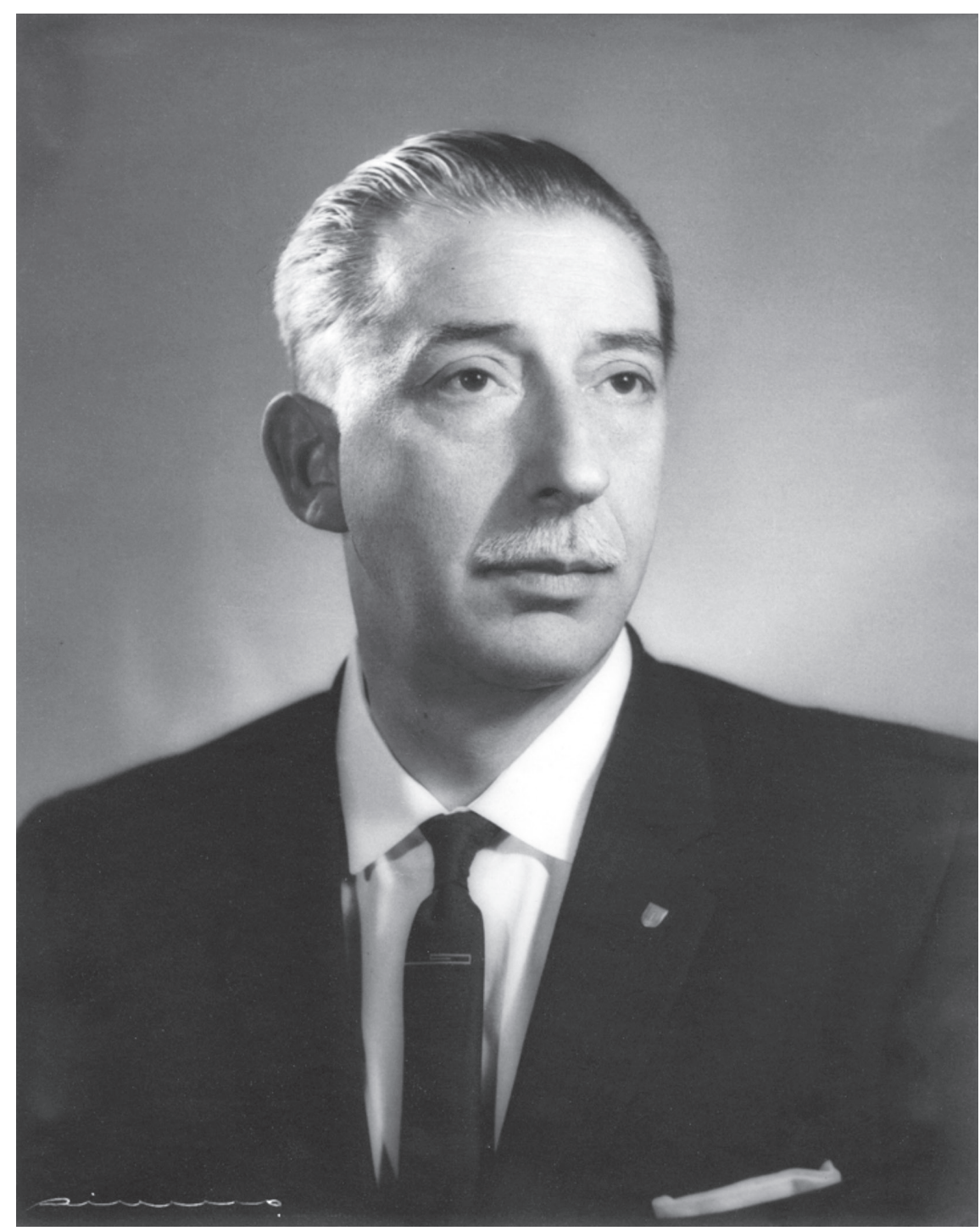

Edgardo Enríquez Frödden, Rector de la Universidad de Concepción (1969 a 1972). Foto gentileza Archivo Fotográfico Universidad de Concepción. 
Reunido el Consejo Superior de la Universidad y de acuerdo al acta del 26 de enero de 1972, "SE ACUERDA, por unanimidad, crear una Escuela de Graduados y de Perfeccionamiento Profesional en la Universidad de Concepción" (Acta Ordinaria Honorable Consejo Superior. Ver Universidad de Concepción, 26 de enero de 1972, s.p.).

Una vez que el Consejo Superior dio el visto bueno para la creación de la Escuela de Graduados, el vicerrector Galo Gómez reunió a la Comisión de Docencia de la Universidad, la cual requirió de algunos informes para poner en marcha la nueva unidad académica. Por ejemplo, la realización de dos encuestas, una de carácter individual a los docentes de la Universidad, en la cual señalaran los estudios que habían realizado tanto en Chile como en el extranjero, los títulos profesionales y los grados académicos que habían obtenido y por último las líneas de investigación que desarrollaban. La segunda encuesta estuvo dirigida a las diferentes unidades académicas, con el propósito de conocer qué asignaturas se dictaban o podían dictarse en cada uno de los departamentos y la proyección de estas asignaturas a los estudios de Posgrado y Perfeccionamiento Profesional.

Una vez obtenidos los resultados de la aplicación de las encuestas, la Comisión de Docencia propuso al Consejo Superior la puesta en marcha de la Escuela de Graduados y Perfeccionamiento Profesional, la cual contaría con un Director y un Secretario Docente.

A partir de lo anterior, la Universidad de Concepción, comprometida con el desarrollo económico y social de la región y el país, pretendía a través de esta instancia contribuir al progreso de la nación por medio del más alto desarrollo que alcanzaran sus docentes e investigadores.

Ocho fueron los objetivos que se planteó la creación de una Escuela de Graduados y Perfeccionamiento Profesional; dentro de estos podemos destacar los siguientes: número uno, "Formar personal altamente calificado, capaz de prestar un servicio adecuado a las exigencias de nuestra realidad social y económica y de fomentar la aplicación de nuevas técnicas en los diferentes campos de la actividad nacional"; el número cuatro, "Fomentar y desarrollar la docencia e investigación multidisciplinaria"; el número siete, "Facilitar y fomentar las interrelaciones a alto nivel entre docencia y científicos regionales, nacionales e internacionales; y el número ocho, "Crear un incentivo interno para la retención de personal altamente calificado en nuestra Universidad y en el país" (Universidad de Concepción, septiembre/ octubre de 1972, p. 6).

Al finalizar su periodo como vicerrector de la Universidad (enero de 1973), el profesor Galo Gómez señaló respecto a la Escuela de Graduados 


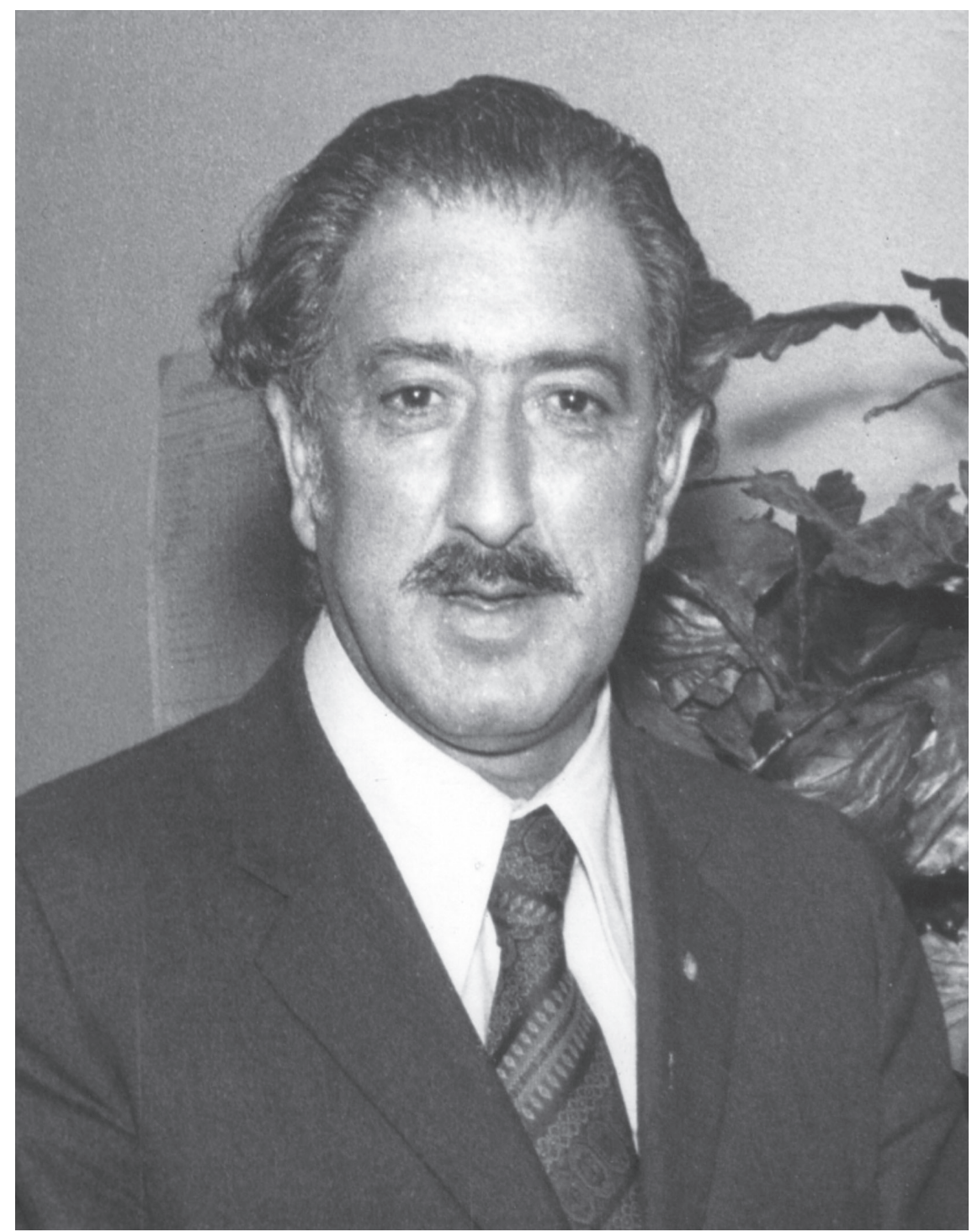

Galo Gómez Oyarzún, Vicerrector Universidad de Concepción (1969 a 1972). Impulsor de la Escuela de Graduados y Perfeccionamiento Profesional entre los años 1971-1972. Foto gentileza Archivo Fotográfico Universidad de Concepción. 
que la tarea inmediata para el año 1973 era contar con un presupuesto propio y un espacio físico para su funcionamiento y la puesta en marcha de la Escuela durante el primer semestre de 1973. A esto agregó que la Universidad de Concepción en sus casi 54 años de vida había alcanzado un nivel de desarrollo y un nivel académico que eran reconocidos nacional e internacionalmente, lo cual la situaba en una perspectiva superior de docencia e investigación. Además, señaló que un importante número de docentes había obtenido grados académicos de Master y Doctor en prestigiosas universidades extranjeras, mientras que otros habían realizado cursos de perfeccionamiento; por lo tanto, se requería aprovechar todas esas capacidades en beneficio de la Universidad y el país. De ahí entonces la necesidad de crear una Escuela de Graduados que contribuyera a profundizar el trabajo científico y se capacitara para una docencia e investigación de alto nivel. Finalmente, Galo Gómez señaló que la Escuela de Graduados de la Universidad de Concepción era la única de su tipo entre todas las universidades chilenas; y ese era uno de sus grandes méritos (Universidad de Concepción, 5 de enero de 1973, p 14).

En aquel contexto la ya aprobada Escuela de Graduados y Perfeccionamiento Profesional debía comenzar sus funciones el año 1973, sin embargo, el golpe de Estado del 11 de septiembre significó una pausa en dicho proceso (Memoria de la Universidad de Concepción, 1972-1973, p. 11).

Si bien Carlos von Plessing -quien se había impuesto como Rector en la elección en noviembre de 1972- había sido cesado de su cargo por parte de la Junta Militar, siguió desempeñando funciones académicas al interior de la Universidad, al punto de que en marzo de 1974, el nuevo Rector Delegado lo designó como Director de la recién creada Escuela de Graduados: "El Rector delegado de la "U” local, Guillermo González Bastías, recibió al mediodía de ayer al profesor Von Plessing, en su oficina. En esta oportunidad Carlos Von Plessing le dio a conocer la decisión de aceptar la dirección de la Escuela de Post grado, la que fue ofrecida en días pasados (ver "Von Plessing dirige...”, 20 de marzo de 1974, p. 7). Acompañarán a Von Plessing, Luis Bardisa U., como Secretario Ejecutivo, y Hernando Urrutia, como Jefe del Departamento de Graduados. Estos tres académicos eran docentes del área de Química y Farmacia.

Años más tarde, von Plessing recordará: "En 1974 se me solicita organizar la Escuela de Graduados de la Universidad. Esto ya había sido creado por el Consejo Superior en 1972; pero el ambiente reinante en esa época no permitió que su organización se concretara" (ver "Ayudando a crecer...", 1991, pp. 10-11). 


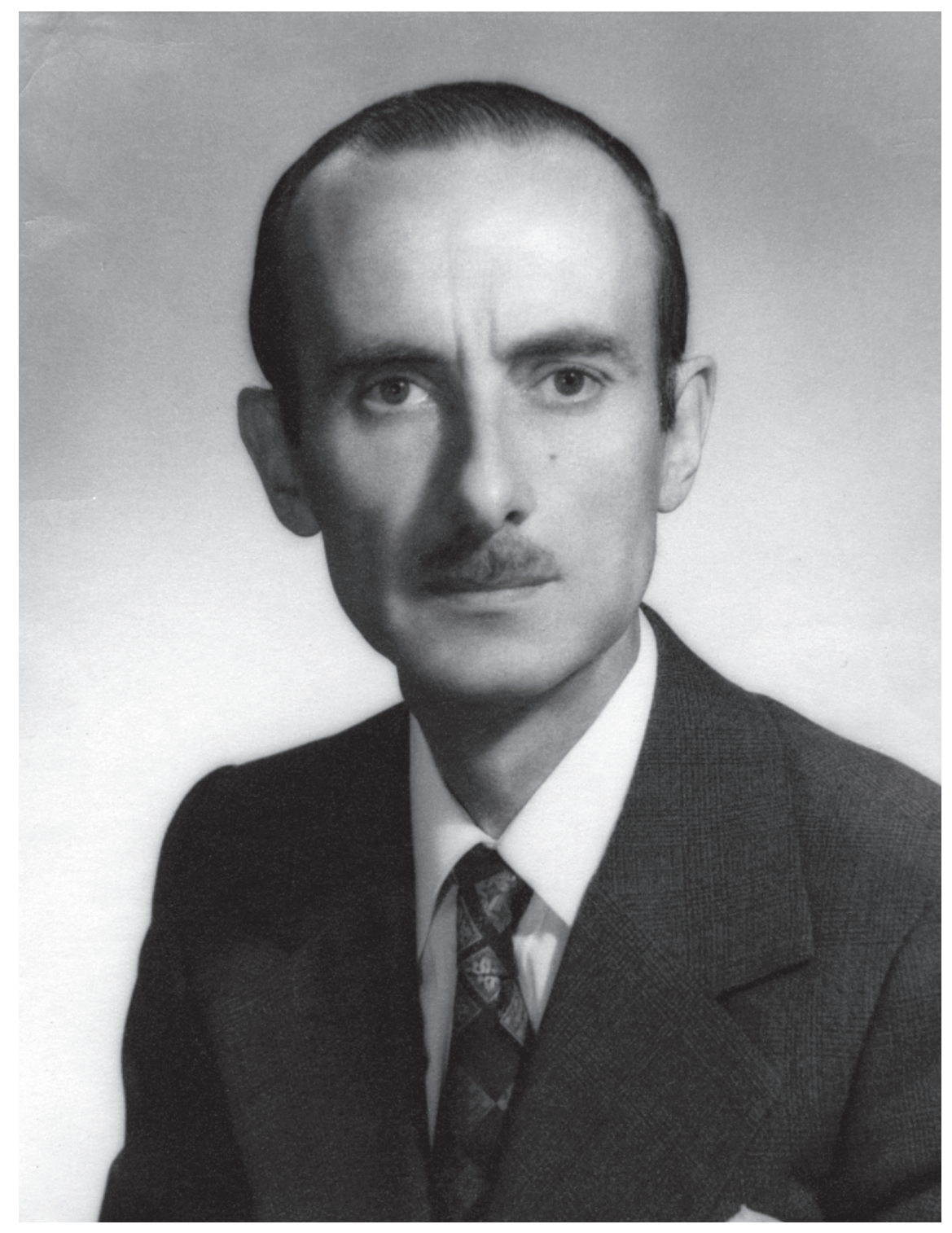

Carlos Von Plessing, Rector Universidad de Concepción (enero a octubre de 1973). Designado primero Director de la Escuela de Graduados en 1974. Foto gentileza Archivo Fotográfico Universidad de Concepción. 


\section{COMENTARIOS FINALES}

La creación de un espacio institucional a cargo de la gestión, administración y coordinación de los estudios de posgrado y perfeccionamiento profesional en la Universidad de Concepción está directamente vinculada a un proceso progresivo de producción de nuevos conocimientos en la casa de estudios, los cuales no se explican sin comprender la importancia histórica que tuvo el apoyo brindado por parte de la institución para que jóvenes docentes viajaran al extranjero a perfeccionar sus conocimientos, sumado a la contratación de destacados académicos e investigadores internacionales, a lo cual se sumó con el tiempo la inquietud intelectual y el impulso profesional para crear cursos de posgrado, hasta llegar a la creación de programas de posgrado y de una Escuela de Graduados, la que llegó a ser en su tiempo, la única de sus características en el país.

Aquel anhelo fue el resultado de un proceso que se fue construyendo a través de los años y que tuvo como aspecto central el apoyar la docencia e investigación al más alto nivel nacional e internacional. De esta forma, la creación de la Escuela de Graduados y Perfeccionamiento Profesional, no fue un hecho meramente coyuntural y menos accidental; todo lo contrario, detrás de la materialización de este proyecto, no solo estuvo el interés de la Universidad, sino también de algunos de sus docentes, investigadores y exalumnos, los cuales consideraban que la institución contaba con todos los elementos para avanzar en esa dirección y así contribuir al desarrollo y progreso del país.

Solamente faltaba la decisión y el compromiso para materializar esto. Fue así como bajo la rectoría de Edgardo Enríquez (1969-1972) y con la coordinación del vicerrector Galo Gómez se tomó la determinación de crear la Escuela de Graduados y Perfeccionamiento Profesional para, de esa forma, dar respuesta a todos aquellos que desde distintos espacios y lugares habían venido trabajando y demandando desde hacía años la creación de una unidad encargada de potenciar el posgrado en la casa de estudios, escenario que se prolonga con éxito hasta nuestros días.

\section{REFERENCIAS}

Ayudando a crecer, Dr. Carlos von Plessing (1991). Tribuna Universitaria 5. Concepción: Universidad de Concepción.

González, I. (1968). Un ensayo de reforma universitaria. Concepción: Universidad de Concepción. 
Una comisión estudiará la creación del doctorado en la Universidad de Concepción (25 de octubre de 1952). La Patria, p. 9.

Universidad de Concepción (11 de mayo de 1959). Reglamento general de los Institutos Generales de la Universidad de Concepción. Archivo Central Universidad de Concepción.

Universidad de Concepción (abril de 1967). Conclusiones de la Convención de Ex Alumnos de la Universidad de Concepción, Concepción. Archivo Central Universidad de Concepción.

Universidad de Concepción (8 de mayo de 1967). Sesión de Directorio. Archivo Central Universidad de Concepción.

Universidad de Concepción (septiembre de 1971). Proyecto de Estructuración de los Estudios de Postgrado en la Universidad de Concepción. Escuela de Graduados y de Perfeccionamiento Profesional. Archivo Central Universidad de Concepción.

Universidad de Concepción (26 de enero de 1972). Acta Ordinaria Honorable Consejo Superior. Archivo Central Universidad de Concepción.

Universidad de Concepción (enero de 1972). Informe para el Consejo Superior Universitario sobre el Proyecto aprobado por las comisiones conjuntas de Docencia y Planeamiento que dice relación con la creación de la Escuela de Graduados y de la Escuela de Perfeccionamiento Profesional de la Universidad de Concepción. Archivo Central Universidad de Concepción.

Universidad de Concepción (septiembre / octubre 1972). Comisión de Docencia del Consejo Superior. Publicación Bimensual, No 10, año III. Archivo Central Universidad de Concepción.

Universidad de Concepción (enero de 1973). La función de la nueva Escuela de Graduados. Boletín del Consejo de Difusión, año 1, número 5.

Universidad de Concepción (1972-1973). Memorias de la Universidad de Concepción.

Von Plessing dirige la Escuela de Postgrado. (miércoles 20 de marzo de 1974). El Diario Color, p. 7. 